\title{
14 \\ From Einstein, Bohr, Schrödinger to Bell and Feynman: a New Quantum Revolution?
}

\begin{abstract}
Alain Aspect
Institut d'Optique, 2 avenue Augustin Fresnel 91120 Palaiseau cedex, France
\end{abstract}

\section{Abstract}

In 1935, the Einstein-Podolsky-Rosen paper introduced a new kind of two-particles quantum state, which was named 'entangled' by Schrödinger. Showing that measurements on both particles were strongly correlated even if the particles were widely separated, Einstein argued that the formalism did not describe all the elements of the physical reality, and he concluded that Quantum Mechanics was not complete. Bohr immediately opposed this reasoning, claiming that Quantum Mechanics was consistent as it was, and that it did not need to be completed, and even that it should not be completed. The discussion between Einstein and Bohr lasted until the death of the two giants, leaving the question unsettled. It was considered by most of the physicists only as an epistemological debate, holding on questions of interpretation, but without any practical consequence on how to use Quantum Mechanics. The situation changed in 1964, when Bell discovered that taking Einstein's point of view led to consequences in contradiction with the predictions of Quantum Mechanics in some (rare) situations. The debate was then displaced from the realm of epistemology to the one of physics, since it could be settled by experiments. After a series of experiments closer and closer to the ideal Gedankenexperiment, more and more physicists realized that entanglement was definitely weirder than any previous concept, and that it might be used for new ways of processing and transmitting information. This prompted the development of a new field, quantum information, which brings us into a new quantum age, and may change our society as dramatically as the first quantum revolution, which gave us integrated circuits and lasers, responsible for the development of the information and communication society. 


\author{
Foreword \\ This chapter is reprinted verbatim from a contribution of the same author \\ with the same title, published in the book Niels Bohr, 1913-2013: Poincaré \\ Seminar 2013, O. Darrigol, B. Duplantier, JM Raimond, V Rivasseau eds., \\ Springer International Publishing (2013).
}

\title{
I. The first quantum revolution: from concepts to technology
}

Five years after the introduction by M. Planck of the quantization of energy exchanges between light and matter [I], A. Einstein took a major step further in 1905, by proposing the quantization of light itself in order to understand the photoelectric effect [2]. It took a decade for this revolutionary hypothesis to be accepted, after R. A. Millikan found experimental evidences in favor of Einstein's hypothesis [3], while Bohr's 1913 model of the atom $[4,5,6]$ gave for the first time a quantitative description of the stability of atoms, and used the relation $\hbar \omega_{i, j}=E_{i}-E_{j}$ to describe emission of absorption of light by atoms.

It took another decade to establish a comprehensive paradigm of Quantum Mechanics, centered about the 1925 formalisms of Heisenberg on the one hand, and Schrödinger on the other. The latter was a wave equation for matter, completing a beautiful duality: like light, matter can behave as either a particle or a wave, elaborating on the original idea of L. de Broglie, while the former relied on the mathematics of matrices. The two formalisms were demonstrated equivalent by Dirac. The success of this formalism was enormous. It became possible to understand mechanical, optical, electrical and thermal properties of matter. In the following decades, Quantum Mechanics allowed physicists to describe particle physics, and to understand amazing properties of matter such as superconductivity or superfluidity. Studies in light-matter interaction were refined by orders of magnitudes, fitting perfectly within the quantum mechanical framework, which had been refined to be applied both in the elementary phenomenon (Quantum Electrodynamics) as well as in complex situations encountered in condensed matter. But in the early 1950's, Quantum Mechanics still appeared as a game to be played by physicists only for the sake of progress in knowledge, without any impact on everyday life.

Half a century later, the applications of quantum physics are all around us in electronics and photonics. The transistor was invented in 1948 by solid-state physicists, after fundamental reflections about the quantum nature of electrical conduction. This invention and its descendants, micro-fabricated integrated circuits, had a monumental impact. Like the steam engine over a century earlier, the transistor changed our lives and gave birth to a new era, the information age. The second technological progeny of Quantum Mechanics is the laser, developed in the late 1950's. Some of its applications are obvious in every day life: bar code readers, CD recorders and players, medical tools, etc. Less visible but perhaps more important is the use of laser light in telecommunications, where it dramatically boosts the flow of information: terabits (millions of millions of information units) per second can be transmitted across the oceans through a single optical fiber. 
Meanwhile, basic research on atom-photon interactions has continued to develop, leading to applications. For example, in 1997 a Nobel Prize was awarded to S. Chu, C. Cohen-Tannoudji, and W. D. Phillips, for the development of methods for cooling and trapping of atoms with lasers. Cold atoms are now used in a new generation of gravimeters, based on atom interferometry, which allow us to explore the underground. Another spectacular application is cold atomic clocks, whose accuracy is now better than $10^{-17}$ (a few seconds error in the age of the universe!). Better clocks will improve the accuracy of the global positioning system (GPS), as well as fast information transfer. Coming full circle, these improved clocks and gravimeters can be applied to fundamental questions, such as tests of general relativity, or the search for slow variation of fundamental physical constants. The first quantum revolution, with its interplay between basic questions and applications, is still at work.

\section{The Einstein-Bohr debate}

\section{I The first Bohr-Einstein debate: single particle Quantum Mechanics}

Quantum mechanics was constructed at the price of several radical and sometimes painful revisions of classical concepts. For instance, to take into account particle-wave duality, Quantum Mechanics had to renounce the idea of a classical trajectory, as stated by the celebrated Heisenberg inequalities. One can also illustrate this renunciation of classical trajectories by remarking that in an interference experiment the particle "follows many paths at once." Such renunciations were so radical that several, including Einstein and de Broglie, could not admit their inevitability, and differed with Bohr who had carved the Rosetta stone of interpretation of the new theory under the name of "Copenhagen interpretation", which was based on the complementarity principle. Einstein did not challenge the formalism and its provisions directly, but seemed to think that the renunciations put forward by Bohr could only signify the incompleteness of the quantum formalism. This position led to Homeric debates in the Solvay congress of 1927 and 1930, when Einstein tried to find an inconsistency in Heisenberg inequalities applied to a single particle, and Bohr each time came with a convincing rebuttal [7].

\subsection{The Einstein-Podolsky-Rosen paper [8]}

In 1935, Einstein raised a completely different objection. Rather than considering the behavior of a single quantum particle, for which the Heisenberg relations state that the position and the velocity cannot be both perfectly defined, Einstein considered two quantum particles, and he discovered that the quantum formalism allowed this pair to be in a quantum state of the form 


$$
\begin{aligned}
\Psi\left(x_{1}, x_{2}\right) & =\int \mathrm{d} p \mathrm{e}^{\frac{i}{\hbar} p\left(x_{1}-x_{2}+x_{0}\right)} \\
& =2 \pi \hbar \delta\left(x_{1}-x_{2}+x_{0}\right) \\
& =\int \mathrm{d} p \mathrm{e}^{\frac{i}{\hbar} p x_{0}} u_{p}\left(x_{1}\right) u_{-p}\left(x_{2}\right),
\end{aligned}
$$

where $u_{p}\left(x_{1}\right)=\exp \left(\mathrm{i} p x_{1} / \hbar\right)$ describes the particle I with a well-defined momentum $p$, while $u_{-p}\left(x_{2}\right)=\exp \left(-\mathrm{i} p x_{2} / \hbar\right)$ describes the particle 2 with a well-defined momentum $-p$.

In such a state, both the momenta of the two particles and their positions are strictly correlated. More precisely, if one measures the position of the first particle and finds $x_{1}$, then the expression of the second line of equation (I) shows that a measurement of the position of particle 2 will yield with certainty the result $x_{2}=x_{1}+x_{0}$. Similarly, the third line shows that if one measures the momentum of the first particle and finds $p$, then a measurement of the momentum of particle 2 will yield with certainty the result $-p$. Since the measurement on the first particle cannot affect the far away second particle, EPR argued, the second particle had already a well-defined position and momentum before any measurement was performed on it. Such a possibility is not envisaged by the standard interpretation of the formalism, for which a measurement of position or momentum on particle 2 can give a priori any result (permitted by equation (I)). EPR concluded that the formalism of Quantum Mechanics is thus incomplete. Moreover, they added, the position and the momentum of the second particle are perfectly defined simultaneously, in contradiction with the Heisenberg uncertainty relation.

\subsection{Schrödinger's reaction [9]}

The reasoning was published in March 1935. As early as August of the same year, Schrödinger sent a paper to the Cambridge Philosophical Society. In his paper, which was communicated by Born, Schrödinger elaborated on the properties of the states of the kind proposed by EPR, which he named "entangled" to emphasize the fact that in such a state, describing two systems that have interacted before separating, the two separated systems "can no longer be described in the same way as before, viz. by endowing each of them with a representative of its own" (i.e. one cannot factorize the wave-function of the whole system as a product of wave functions describing each system separately). He then formulated in his own way the argument of EPR, calling its conclusion 'a paradox'.' And he made it clear that "[his] paper does not aim at a solution of the paradox, it rather adds to it, if possible". In conclusion, he pointed out the role of the timing in the 'paradox': "The paradox would be shaken, though, if an observation did not relate to a definite moment. But this would make the present interpretation of Quantum Mechanics meaningless, because ... the objects of its predictions are considered to be the results of measurements for definite moments of time." We will see later that indeed the contradiction, between Quantum Mechanics and the intuitive concept of physical reality advocated by Einstein, happens for measurements that are space-like separated, a situation possible only because measurements are done at a well-defined position and time.

(I) It seems that it is Schrödinger who introduced first the term 'paradox', a word not employed by EPR. 


\subsection{Bohr's rebuttal [10]}

According to witness, Bohr was shattered by the EPR paper. His reaction has been described by his grandson: ${ }^{2}$

"When the EPR paper was published, Niels Bohr was working with Leon Rosenfeld on the problem of the measurability of electric and magnetic fields in Quantum Mechanics. Rosenfeld told the story as follows:

This onslaught came down upon us as a bolt from the blue. Its effect on Bohr was remarkable.... As soon as Bohr had heard my report of Einstein's argument, everything else was abandoned: we had to clear up such a misunderstanding at once. We should reply by taking up the same example and showing the right way to speak about it. In great excitement, Bohr immediately started dictating to me the outline of such a reply. Very soon, however, he became hesitant: "No, this won't do, we must try all over again ... we must make it quite clear ..." ... Eventually, he broke off with the familiar remark that he "must sleep on it". The next morning he at once took up the dictation again, and I was struck by a change of the tone in the sentences: there was no trace in them of the previous day's sharp expressions of dissent. As I pointed out to him that he seemed to take a milder view of the case, he smiled: "That's a sign", he said, "that we are beginning to understand the problem." Bohr's reply was that yes, nature is actually so strange. The quantum predictions are beautifully consistent, but we have to be very careful with what we call 'physical reality'."

The reply of Bohr [10] was published after no more than four months, in Physical Review, with the same title as the EPR paper: "Can Quantum Mechanical Description of Physical Reality be Considered Complete?". In contrast with Schrödinger, Bohr refuted the reasoning of EPR, on the basis of the notion of complementarity. More precisely, after recalling that when one considers one particle one must choose between measuring the position or the momentum of that particle, he emphasizes similarly that in the EPR situation one has to choose the observable one wants to measure on the first EPR particle, and it is only the value of that observable that can be known with certainty for the second particle.

Actually, Bohr's reply to EPR was for him an opportunity for many statements, with carefully chosen wording, about the specifity of the quantum mechanical description of the world, by comparison with the classical description. Here are a few excerpts of [10]:

- The apparent contradiction in fact discloses only an essential inadequacy of the customary viewpoint of natural philosophy for a rational account of physical phenomena of the type with which we are concerned in quantum mechanics.

(2) Tomas Bohr, speech at the Carlsberg Academy for the Niels Bohr Gold Medal 2013 Ceremony. 
- Indeed the finite interaction between object and measuring agencies conditioned by the very existence of the quantum of action entails - because of the impossibility of controlling the reaction of the object on the measuring instruments if these are to serve their purpose - the necessity of a final renunciation of the classical ideal of causality and a radical revision of our attitude towards the problem of physical reality.

- In the phenomena concerned we are not dealing with an incomplete description characterized by the arbitrary picking out of different elements of physical reality at the cost of sacrifying other such elements, but with a rational discrimination between essentially different experimental arrangements and procedures which are suited either for an unambiguous use of the idea of space location, or for a legitimate application of the conservation theorem of momentum.

- Any comparison between Quantum Mechanics and ordinary statistical mechanics, however useful it may be for the formal presentation of the theory, is essentially irrelevant. Indeed we have in each experimental arrangement suited for the study of proper quantum phenomena not merely to do with an ignorance of the value of certain physical quantities, but with the impossibility of defining these quantities in an unambiguous way.

- From our point of view we now see that the wording of the above-mentioned criterion of physical reality proposed by Einstein, Podolsky and Rosen contains an ambiguity as regards the meaning of the expression "without in any way disturbing a system." Of course, there is in a case like that just considered no question of a mechanical disturbance of the system under investigation during the last critical stage of the measuring procedure. But even at this stage there is essentially the question of an influence on the very conditions which define the possible types of predictions regarding the future behavior of the system. Since these conditions constitute an inherent element of the description of any phenomenon to which the term "physical reality" can be properly attached, we see that the argumentation of the mentioned authors does not justify their conclusion that quantum-mechanical description is essentially incomplete. On the contrary this description, as appears from the preceding discussion, may be characterized as a rational utilization of all possibilities of unambiguous interpretation of measurements, compatible with the finite and uncontrollable interaction between the objects and the measuring instruments in the field of quantum theory. In fact, it is only the mutual exclusion of any two experimental procedures, permitting the unambiguous definition of complementary physical quantities, which provides room for new physical laws, the coexistence of which might at first sight appear irreconcilable with the basic principles of science. It is just this entirely new situation as regards the description of physical phenomena that the notion of complementarity aims at characterizing.

The last quotation is representative of Bohr's reply. It is not so much a reply to EPR, than a statement about complementarity being the most important feature of Quantum Mechanics, allowing that theory to render an account of phenomena that were impossible to describe in classical physics. 
In contrast to the discussions about the Heisenberg inequalities for a single particle, Bohr's reply is not a technical refutation of Einstein's reasoning, it is rather an elaboration on an epistemological point of view, totally opposed to the one of Einstein. This may be a major reason for the lack of interest shown by most physicists about that debate: why should active physicists bother about such a discussion, if Bohr and Einstein disagreed only about the interpretation of the formalism, but not about the formalism itself? Indeed, Einstein and Bohr did not disagree on the results of the calculation, but on the conclusion to draw about the need or the possibility to complete that formalism.

The discussion between Bohr and Einstein on that subject continued until the end of their lives, without any significant evolution of their positions.

\section{Bohm's version of the EPR reasoning}

In his excellent book on Quantum Mechanics [II], David Bohm presented a version of the EPR situation that differed significantly from the original one (see also [12]). Indeed, Bohm's scheme is based on spins $1 / 2$, whose observables are dichotomic, rather than particles moving in space, whose position and momentum are continuous observables. This scheme had far reaching consequences. Firstly, it allows one to give a definitely simpler presentation of the EPR argument. Secondly and yet more important, it allowed Bell to discover his famous inequalities [13]. Actually, as it is now clear, it is only with dichotomic variables that one has Bell's type inequalities that can be tested directly, such as the celebrated CHSH inequalities [14], while continuous variables do not permit such simple tests [15].

\section{I Bohm's Gedankenexperiment with photons}

Rather than considering spin I/2 particles, whose spin components are measured with Stern-Gerlach analyzers, we consider an equivalent scheme with photons, whose polarizations are measured with linear polarization analyzers. The situations are equivalent since in both cases we have dichotomic observables, whose measurement can give only two possible results: parallel or perpendicular to the polarization analyzer orientation in the case of a photon; aligned or anti-aligned with the SternGerlach analyzer orientation in the case of spin $1 / 2$.

The optical variant of the Bohm's version of the E.P.R. Gedankenexperiment is shown on figure I. A source $S$ emits a pair of photons $\left(v_{1}, v_{2}\right)$ with different frequencies, counterpropagating along $\mathrm{Oz}$. Suppose that the polarization part of the state vector describing the pair is:

$$
\left|\Psi\left(\nu_{1}, \nu_{2}\right)\right\rangle=\frac{1}{\sqrt{2}}\{|x, x\rangle+|y, y\rangle\}
$$

where $|x\rangle$ and $|y\rangle$ are linear polarizations states. This state is remarkable: it cannot be factorized into a product of two states associated to each photon, so we cannot ascribe 
any well-defined polarization state to each individual photon. Such a state describing a system of several objects in a situation that can only be described globally, is an entangled state. Moreover, we notice that the state (2) is rotationally invariant, and can be written as

$$
\left|\Psi\left(\nu_{1}, \nu_{2}\right)\right\rangle=\frac{1}{\sqrt{2}}\left\{\left|x^{\prime}, x^{\prime}\right\rangle+\left|y^{\prime}, y^{\prime}\right\rangle\right\}
$$

where the $x^{\prime}$ and $y^{\prime}$ axes are any set of orthogonal axes, perpendicular to the $z$-axis. We can perform linear polarization measurements on each of the two photons, with analyzers I and II. The analyzer I, in orientation a, is followed by two detectors, giving results + or - , corresponding to a linear polarization found parallel or perpendicular to $\mathbf{a}$. The analyzer II, in orientation $\mathbf{b}$, acts similarly. If the direction of analysis is along $x$, the results + and - are respectively associated with the polarizations $|x\rangle$ and $|y\rangle$ (note the technical difference with spin I/2 measurements, where the two 'orthogonal' results associated with a measurement along $x$ correspond to two components along $x$ and $-x$ ).

\subsection{EPR reasoning with photons}

We can repeat the EPR reasoning, using the state (2). We consider first a linear polarization measurement on photon $v_{1}$, along the $x$-axis. If we find + , which is the result associated with $|x\rangle$, we are sure that a measurement on $v_{2}$ along the same direction would also give + , and similarly for results - associated with $|y\rangle$. Measuring $v_{1}$ along $x$ allows us to predict with certainty what we would obtain for a similar measurement on $v_{2}$.

Let us now consider measurements along $x$. Since the state $\left|\Psi\left(v_{1}, v_{2}\right)\right\rangle$ can be written as in equation (3), it is clear that if a measurement on $v_{1}$ yields + , which is the result associated with $\left|x^{\prime}\right\rangle$, then a similar measurement on $v_{2}$ along $x^{\prime}$ would also give + , and similarly for results - associated with $\left|y^{\prime}\right\rangle$. Measuring $v_{1}$ along $x^{\prime}$ also allows us to predict with certainty what we would obtain for a similar measurement on $v_{2}$.

Since the measurement on the first photon cannot affect the far away second photon, EPR would argue, the second photon had already well-defined components of polarizations along $x$ and along $x$, before any measurements was performed on it. Such a statement is in contradiction with the fact that in the state $\left|\Psi\left(v_{1}, v_{2}\right)\right\rangle$ a measurement on photon $v_{2}$ can give results + or - with equal probabilities. One can then conclude that the formalism of Quantum Mechanics is not complete.

Moreover, EPR would add, the values of the polarization of $v_{2}$ are well-defined simultaneously along $x$ and $x$, although the corresponding observables do not commute (if the two axes are neither parallel nor orthogonal). This is in contradiction with the Heisenberg uncertainty relations. 


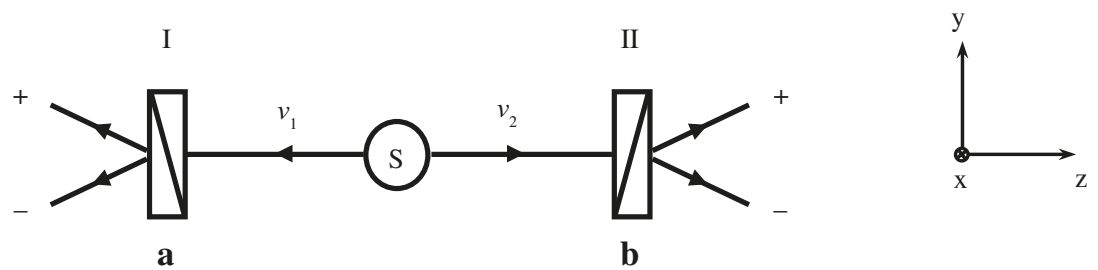

Figure 1. Einstein-Podolsky-Rosen-Bohm Gedankenexperiment with photons. The two photons $v_{1}$ and $v_{2}$, emitted in the state of equation (2), are analyzed by linear polarizers in orientations defined by unit vectors $\mathbf{a}$ and $\mathbf{b}$ perpendicular to the z-axis. Each photon is eventually detected either in the output channel + or in the output channel - of the polarizer where it enters. One can measure the probabilities of single or joint detections in the output channels of the polarizers.

\subsection{Discussion}

Reasoning on the Bohm's version of the EPR Gedankenexperiment allows us to emphasize a point that was already implicitly present in the original EPR paper, but not as clearly. There is no need to consider two non commuting observables on one of the particles, to conclude that Quantum Mechanics must be completed, since it does not render an account of all the 'elements of the physical reality'. Let us recall the EPR definition of 'elements of the physical reality": "If, without in any way disturbing a system, we can predict with certainty (i.e., with probability equal to unity) the value of a physical quantity, then there exists an element of physical reality corresponding to this physical quantity." According to EPR, a measurement on $v_{1}$ cannot disturb $v_{2}$, which is far away (implicitly space-like separated), and nevertheless we can infer with certainty the result of a measurement on $v_{2}$ along $x$, if we do it. So there is a corresponding element of reality, which is not present in the state (2), which does not predict a precise value for the measurement on $v_{2}$.

The fact that such a reasoning can be repeated for another direction of analysis, associated with a non commuting observable, is a supplementary element, which was not necessary to conclude to the incompleteness of Quantum Mechanics. But this fact will play a crucial role in the violation of Bell's inequalities by quantum predictions, and this is why we give now the results of a standard quantum mechanical calculation for polarizers at any orientation.

\subsection{Correlations: general case}

It is easy to derive the quantum mechanical predictions for the measurements of polarization, single or in coincidence, for polarizers in any orientations $\mathbf{a}$ and $\mathbf{b}$. For the state of equation (2), a simple calculation yields the probabilities of single detections

$$
\begin{aligned}
& P_{+}(\mathbf{a})=P_{-}(\mathbf{a})=\frac{1}{2} \\
& P_{+}(\mathbf{b})=P_{-}(\mathbf{b})=\frac{1}{2} .
\end{aligned}
$$


Each individual polarization measurement gives a random result.

The probabilities of joint detections are easily obtained by projecting the two photon state (2) onto the four state vectors $\left| \pm_{\mathrm{a}}, \pm_{\mathrm{b}}\right\rangle$ associated with the four possible joint results $( \pm, \pm$ )

$$
\begin{aligned}
& P_{++}(\mathbf{a}, \mathbf{b})=P_{--}(\mathbf{a}, \mathbf{b})=\frac{1}{2} \cos ^{2}(\mathbf{a}, \mathbf{b}) \\
& P_{+-}(\mathbf{a}, \mathbf{b})=P_{-+}(\mathbf{a}, \mathbf{b})=\frac{1}{2} \sin ^{2}(\mathbf{a}, \mathbf{b})
\end{aligned}
$$

If we consider the cases where the polarizers I and II are parallel, i.e. $\mathbf{a}=\mathbf{b}$, we have

$$
\begin{aligned}
& P_{++}(\mathbf{a}, \mathbf{b})=P_{--}(\mathbf{a}, \mathbf{b})=\frac{1}{2} \\
& P_{+-}(\mathbf{a}, \mathbf{b})=P_{-+}(\mathbf{a}, \mathbf{b})=0 .
\end{aligned}
$$

This is in agreement with our reasoning of subsection 3.2: a measurement on $v_{1}$ along $x$ can give either the result + or the result - , but if one gets + then the measurement on $v_{2}$ along the same direction $x$ will give with certainty the result + (the conditional probability is I). The same reasoning holds for any direction.

A convenient way to measure the amount of correlations between classical random quantities, such as the results of measurements by polarizers I or II, is to calculate the classical correlation coefficient. For the polarization measurements considered above, whose average values are 0 (see equations (4)), it is defined as

$$
E(\mathbf{a}, \mathbf{b})=P_{++}(\mathbf{a}, \mathbf{b})+P_{--}(\mathbf{a}, \mathbf{b})-P_{+-}(\mathbf{a}, \mathbf{b})-P_{+-}(\mathbf{a}, \mathbf{b}) .
$$

Using (5), we find a correlation coefficient predicted by Quantum Mechanics

$$
E_{\mathrm{QM}}(\mathbf{a}, \mathbf{b})=\cos (\mathbf{a}, \mathbf{b})
$$

In the particular case of parallel polarizers $(\mathbf{a}, \mathbf{b})$, we find $E_{\mathrm{QM}}(\mathbf{a}, \mathbf{b})=1$ : this confirms that the correlation is total. In conclusion, the quantum mechanical calculations shows that although each individual measurement gives random results, these random results are correlated, as expressed by equation (8). 


\section{How to complete Quantum Mechanics? Supplementary parameters}

\section{I What is suggested by the quantum formalism is weird}

A most natural approach to find a way to complete the quantum formalism may seem to follow the quantum mechanical calculations leading to (5). In fact, there are several ways to do this calculation. The most direct one is to project the two photon state (2) onto the four state vectors $\left| \pm_{\mathrm{a}}, \pm_{\mathrm{b}}\right\rangle$ associated with the four possible joint results $( \pm, \pm)$. However, this calculation bears on state vectors of the configuration space of the two particles, and it is not clear how to deduce elements of the physical reality pertaining to the ordinary space, an implicit demand of EPR.

In order to overcome this problem, and find a description in our ordinary space, we treat separately the two measurements done on both ends of the experiment, and we split the joint measurement in two steps. Suppose for instance that the measurement on photon $v_{1}$ takes place first and gives the result + , with the polarizer I aligned along $x$. To proceed with the following of the calculation, we must then use the postulate of reduction of the state vector, which states that after this measurement, the new state vector describing the pair is obtained by projection of the initial state vector, onto the eigenspace associated to the result + : this two dimensional eigenspace has a basis $\{|x, x\rangle ;|x, y\rangle\}$. Using the corresponding projector, we find

$$
\left|\Psi^{\prime}\left(\nu_{1}, \nu_{2}\right)\right\rangle=|x, x\rangle
$$

This means that immediately after the first measurement, photon $v_{1}$ falls into the polarization state $|x\rangle$ : this was to be expected because it was measured with a polarizer oriented along $x$, and the result + was found. More surprisingly, the distant photon $v_{2}$, which has not yet interacted with any polarizer, has also been projected into the state $|x\rangle$ with a well-defined polarization, parallel to the one found for photon $v_{1}$.

This surprising conclusion, however, leads to the correct final values (5) for the probabilities of joint detections, by a straightforward use of Malus law in the case where the subsequent measurement on photon $v_{2}$ is done along a different direction.

The calculation in two steps therefore gives the same result as the direct calculation. But in addition it suggests the following picture in the ordinary space:

- Photon $v_{1}$, which had not a well-defined polarization before its measurement, takes the polarization associated to the obtained result, at the moment of its measurement: this is not surprising.

- When the measurement on $v_{1}$ is done, photon $v_{2}$, which had not a well-defined polarization before this measurement, is projected into a state of polarization parallel 
to the result of the measurement on $v_{1}$. This is very surprising, because this change in the description of $v_{2}$ happens instantaneously, whatever the distance between the two photons at the moment of the first measurement, and without any effective measurement being done on photon $v_{2}$.

This picture seems in contradiction with relativity. According to Einstein, what happens in a given region of space-time cannot be influenced by an event happening in a region of space-time that is separated by a space-like interval. It is therefore not unreasonable to try to find more acceptable descriptions of the EPR correlations. It is such a picture that we consider now.

\subsection{Supplementary parameters}

Correlations between distant measurements on two separated systems that had previously interacted are common in the classical world. For instance, if a mechanical object with a null angular momentum is split in two parts by some internal repulsion, the angular momenta of the two separated parts remain equal and opposite in the case of a free evolution. In the general case where each fragment is submitted to some external action that may evolve its angular momentum, the two momenta remain nevertheless correlated, since their values are at each moment determined by their initial values, which had a perfectly defined sum.

It is tempting to use such a classical picture to render an account of the EPR correlations, in term of common properties of the two systems. Let us consider again the perfect correlation of polarization measurements in the case of parallel polarizers along $x$. We can easily describe the observed correlations by assuming that some pairs are emitted with both photons in a well-defined polarization along $x$, while other pairs are emitted with both photons in a well-defined polarization perpendicular to $x$. If the proportions of the two kinds of pairs are equal, we obtain the total correlation predicted by Quantum Mechanics.

Using such a model for describing such correlations is good scientific methodology. Indeed, when biologists or medical doctors observe strong correlations between some feature of identical twins, they can conclude safely that this feature is determined by the identical sets of chromosomes. We are thus led to admit that there is some common property ('an element of physical reality'), whose value determines the result of the polarization. But such a property, which may differ from one pair to another one, is not taken into account by the quantum mechanical state vector (2), which is the same for all pairs. One can thus conclude with EPR that Quantum Mechanics is not complete. And this is why such additional properties are referred to as "supplementary parameters", or "hidden-variables".

As a conclusion, it seems possible to describe the EPR correlations by such a picture, involving supplementary parameters that differ from one pair to another one. It can be hoped to recover the statistical quantum mechanical predictions when averaging over the supplementary parameters, which amounts to considering Quantum Mechanics as the Statistical Mechanics description of a deeper level. It seems that so was Einstein's position [12, 16, 17]. In contrast, we already mentioned that Bohr strongly refused such an option ('any comparison between Quantum Mechanics and ordinary statistical mechanics 
- however useful it may be for the formal presentation of the theory - is essentially irrelevant'). Note that at this stage of the reasoning, a commitment to Einstein's position does not contradict Quantum Mechanics: in fact, Einstein never suggested that the predictions of Quantum Mechanics were wrong, he considered that it was not the deepest level of description that could be used.

\section{Bell's inequalities}

\section{I Formalism}

Three decades after the EPR paper, Bell translated into (quite simple) mathematics the consequences of the preceding discussion, and he explicitly introduced supplementary parameters, denoted $\lambda$. Their distribution on an ensemble of emitted pairs is specified by a probability distribution $\rho(\lambda)$, such that

$$
\begin{aligned}
& \rho(\lambda) \geq 0, \\
& \int d \lambda \rho(\lambda)=1 .
\end{aligned}
$$

For a given pair, characterized by a given supplementary parameter $\lambda$, the results of measurements at polarizers I and II are given by the bi-valued functions

$$
\begin{aligned}
& A(\lambda, \mathbf{a})= \pm 1 \\
& B(\lambda, \mathbf{a})= \pm 1 .
\end{aligned}
$$

A particular Supplementary Parameter Theory is completely defined by the explicit form of the functions $\rho(\lambda), A(\lambda, \mathbf{a})$ and $B(\lambda, \mathbf{b})$. It is then easy to express the probabilities of the various results of measurements, and the correlation function assumes the simple form

$$
E(\mathbf{a}, \mathbf{b})=\int d \lambda \rho(\lambda) A(\lambda, \mathbf{a}) B(\lambda, \mathbf{b})
$$

\subsection{A (naive) example of Supplementary Parameters Theory}

As an example of Supplementary Parameter Theory, we present a model where each photon traveling along $z$ is supposed to have a well-defined linear polarization, perpendicular to the $z$-axis, determined by its angle $\lambda$ with the $x$-axis. In order to account for the strong correlation, we assume that the two photons of a same pair are emitted with the same 


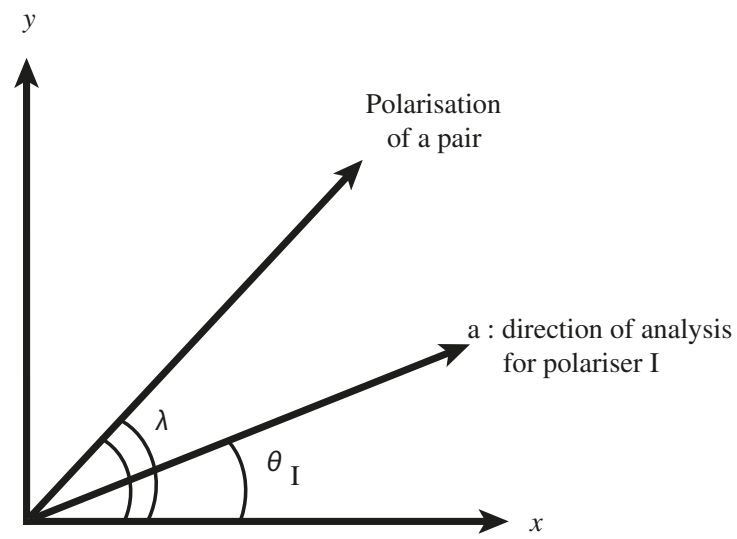

Figure 2. The naive example of Supplementary Parameters Theory. Each pair of photons has a 'direction of polarisation', defined by $\lambda$, which is the supplementary parameter of the model. Polarizer I makes a polarization measurement along $\boldsymbol{a}$, at an angle $\theta_{I}$ from the $x$-axis.

linear polarization, defined by the angle $\lambda$ (figure 2). The polarization of the various pairs is randomly distributed, according to a probability distribution that we take rotationally invariant:

$$
\rho(\lambda)=\frac{1}{2 \pi}
$$

We must also give an explicit form for the functions $A(\lambda, \mathbf{a})$ and $B(\lambda, \mathbf{b})$, which we take with the following form

$$
\begin{aligned}
& A(\lambda, \mathbf{a})=\operatorname{sign}\left\{\cos 2\left(\theta_{\mathrm{I}}-\lambda\right)\right\} \\
& B(\lambda, \mathbf{b})=\operatorname{sign}\left\{\cos 2\left(\theta_{\mathrm{II}}-\lambda\right)\right\},
\end{aligned}
$$

where the angles $\theta_{\mathrm{I}}$ and $\theta_{\mathrm{II}}$ indicate the orientations of the polarisers. Note that these forms are very reasonable: $A(\lambda, \mathbf{a})$ assumes the value $+I$ when the polarization of photon $v_{1}$ is closer to the direction of analysis a, and $-I$ for the complementary case (polarization closer to the perpendicular to a). With this explicit model, we can calculate the probabilities of the various measurements, and the polarization correlation function. We find inserting the expressions $(13,14)$ into $(12)$ :

$$
E(\mathbf{a}, \mathbf{b})=1-4 \frac{\left|\theta_{\mathrm{I}}-\theta_{\mathrm{II}}\right|}{\pi}=1-4 \frac{|(\mathbf{a}, \mathbf{b})|}{\pi}
$$




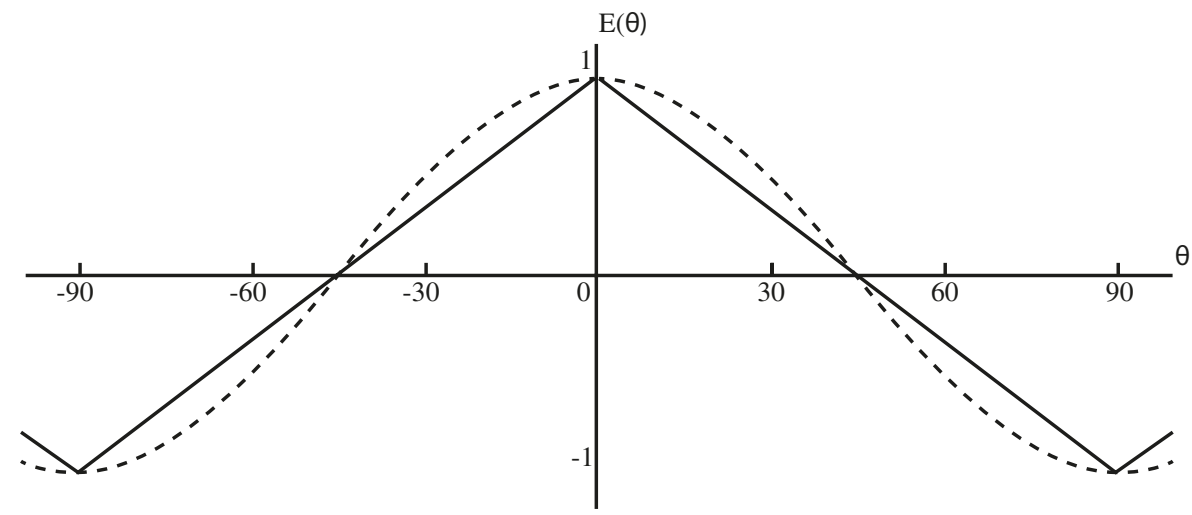

Figure 3. Polarization correlation coefficient, as a function of the relative orientation of the polarisers: (i) Dotted line: quantum mechanical prediction; (ii) solid line: the naive supplementary parameters model.

This is a remarkable result. Note first that $E(\mathbf{a}, \mathbf{b})$ depends only on the relative angle $(\mathbf{a}, \mathbf{b})$, as the quantum mechanical prediction (8). Moreover, as shown on figure 3, the difference between the predictions of the supplementary parameters model and the quantum mechanical predictions is not large, and the agreement is exact for the angles 0 and $\pi / 2$, i.e., the cases of total correlation. This result, obtained with an extremely simple supplementary parameters model, is very encouraging, and it might be hoped that a more sophisticated model could be able to reproduce exactly the quantum mechanical predictions. Bell's discovery is the fact that the search for such models is hopeless, as we are going to show now.

\subsection{Bell's inequalities}

There are many different forms, and demonstrations of Bell's inequalities. We give here a very simple demonstration leading to a form directly applicable to the experiments. Let us consider the quantity

$$
\begin{gathered}
s=A(\lambda, \mathbf{a}) \cdot B(\lambda, \mathbf{b})-A(\lambda, \mathbf{a}) \cdot B\left(\lambda, \mathbf{b}^{\prime}\right)+A\left(\lambda, \mathbf{a}^{\prime}\right) \cdot B(\lambda, \mathbf{b})+A\left(\lambda, \mathbf{a}^{\prime}\right) \cdot B\left(\lambda, \mathbf{b}^{\prime}\right) \\
=A(\lambda, \mathbf{a})\left[B(\lambda, \mathbf{b})-B\left(\lambda, \mathbf{b}^{\prime}\right)\right]+A\left(\lambda, \mathbf{a}^{\prime}\right)\left[B(\lambda, \mathbf{b})+B\left(\lambda, \mathbf{b}^{\prime}\right)\right] .
\end{gathered}
$$

Remembering that the four quantities $A$ and $B$ take only the values $\pm I$, a simple inspection of the second line of (16) shows that

$$
s\left(\lambda, \mathbf{a}, \mathbf{a}^{\prime}, \mathbf{b}, \mathbf{b}^{\prime}\right)= \pm 2 .
$$


The average $S\left(\mathbf{a}, \mathbf{a}^{\prime}, \mathbf{b}, \mathbf{b}^{\prime}\right)$ of $s\left(\lambda, \mathbf{a}, \mathbf{a}^{\prime}, \mathbf{b}, \mathbf{b}^{\prime}\right)$ over $\lambda$ is therefore comprised between +2 and 2. Recalling (I2), we thus conclude that

$$
-2 \leq S\left(\mathbf{a}, \mathbf{a}^{\prime}, \mathbf{b}, \mathbf{b}^{\prime}\right) \leq+2
$$

where

$$
S\left(\mathbf{a}, \mathbf{a}^{\prime}, \mathbf{b}, \mathbf{b}^{\prime}\right)=E(\mathbf{a}, \mathbf{b})-E\left(\mathbf{a}, \mathbf{b}^{\prime}\right)+E\left(\mathbf{a}^{\prime}, \mathbf{b}\right)+E\left(\mathbf{a}^{\prime}, \mathbf{b}^{\prime}\right) .
$$

These are the $\mathrm{BCHSH}$ inequalities, i.e., Bell's inequalities as generalized by Clauser, Horne, Shimony and Holt [14]. They bear upon the combination $S$ of the four polarization correlation coefficients, associated with two directions of analysis for each polarizer. They apply to any Supplementary Parameter Theory of the very general form defined in subsection 5.1 , of which our naive model is only an example.

\section{Conflict with Quantum Mechanics}

\section{I Evidence of a conflict}

Let us consider the sets of orientations shown in figure 4.a. If we express the quantity $S\left(\mathbf{a}, \mathbf{a}, \mathbf{b}, \mathbf{b}^{\prime}\right)$ of equation (19) with the correlation coefficients (8) predicted by Quantum Mechanics for the EPR state, we find

$$
S_{\mathrm{QM}}\left(\mathbf{a}, \mathbf{a}^{\prime}, \mathbf{b}, \mathbf{b}^{\prime}\right)=2 \sqrt{2}
$$

a value obviously in conflict with the right inequality in (18). We have thus found a situation where the quantum mechanical predictions cannot be reproduced (mimicked) by Supplementary Parameters Theories. This is the essence of Bell's theorem: it is impossible to find a Supplementary Parameter Theory, of the general form defined in subsection 5.I, able to reproduce all the predictions of Quantum Mechanics. This statement is the generalization of what appears on figure 3 , for the particular supplementary parameter model considered in subsection 5.2: the model exactly reproduces the predictions of Quantum Mechanics for some particular angles $(0, \pi / 4, \pi / 2)$, but it somewhat deviates at other angles. The importance of Bell's theorem is that it is not restricted to a particular supplementary parameters model, but it is general. 

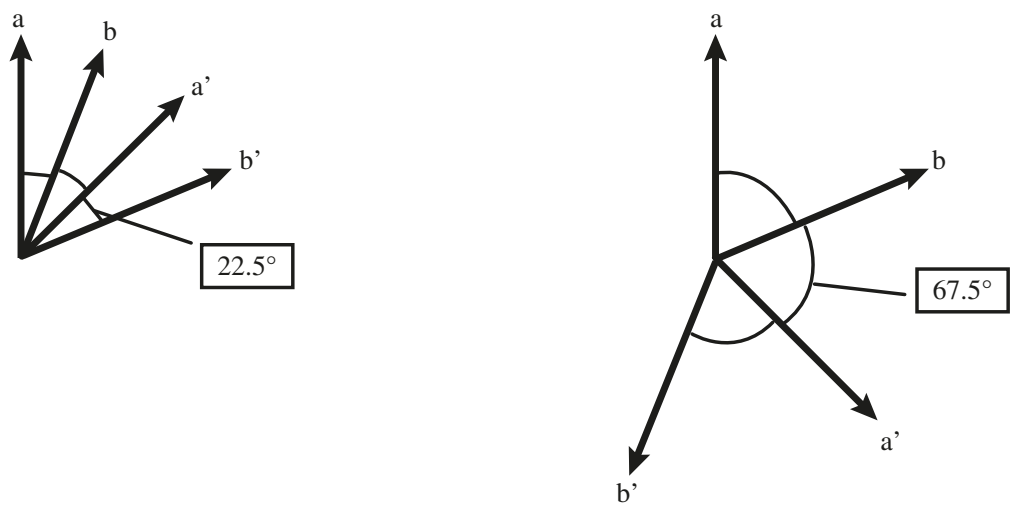

Figure 4. Orientations yielding the largest conflict between Bell's inequalities and Quantum Mechanics.

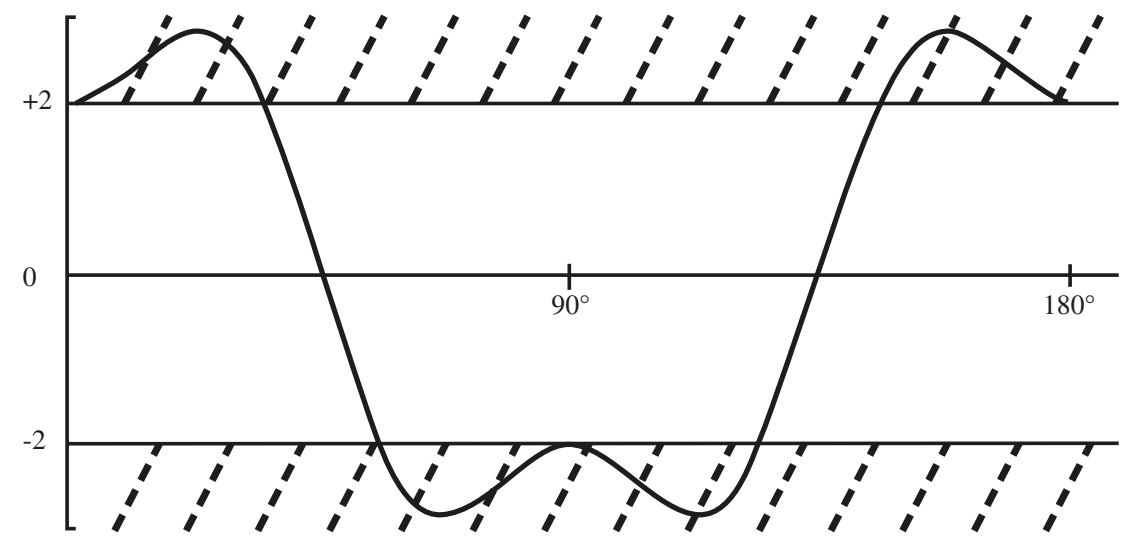

Figure 5. $S_{Q M}(\mathbf{a}, \mathbf{a}, \mathbf{b}, \mathbf{b})$ as predicted by Quantum Mechanics for EPR pairs, for the condition $(\mathbf{a}, \mathbf{b})=(\mathbf{b}, \mathbf{a})=\left(\mathbf{a}^{\prime}, \mathbf{b}^{\prime}\right)=\theta$, which maximizes the conflict with Bell's inequalities. The conflict happens when $|S|$ is larger than 2, and it is maximum for the sets of orientations of figure 4.

It is possible to show that in fact the orientations of figure 4 lead to the largest conflict. Moreover, as shown on figure 5, there are many orientations where there is no conflict between the quantum mechanical predictions and Bell's inequalities. Recalling the EPR discussion, it is interesting to remark that the quantum mechanical predictions do not violate Bell's inequalities if one considers only orientations associated to observables that commute. 


\subsection{Discussion: the locality condition}

Having a conflict between a very reasonable model, in the spirit of Einstein's world views, and Quantum Mechanics, a so successful theory, clearly cries for a discussion to try to identify the properties of the model that entail the conflict. We therefore examine now the various ingredients underlying the Supplementary Parameter Theories introduced in subsection 4.I.

A first ingredient is the use of supplementary parameters, carried along by each particle, and determining the outcome of the measurement at each measuring apparatus. As we have seen, supplementary parameters have been introduced in order to render an account of the correlations at a distance, without invoking instantaneous action at a distance. The model is thus strongly related to a conception of the world, as expressed by Einstein, and sometimes called 'local realism', where it is meaningful to invoke separated physical realities for space-like separated systems. It is clear that here the word 'local' must be understood in the relativistic sense of 'localized in space time', not to be confused with Bell's locality condition that we will encounter below.

Another apparent ingredient of the formalism is determinism. As a matter of fact, in the formalism of subsection 4 .I the results $A(\lambda, \mathbf{a})$ and $B(\lambda, \mathbf{b})$ of the polarization measurements are certain, once $\lambda$ is fixed. One has speculated that it may be the reason for a conflict with the non-deterministic formalism of Quantum Mechanics. In fact, as first shown by Bell, and subsequently elaborated, ${ }^{3}$ it is easy to generalize the formalism of subsection 4.I to Stochastic Supplementary Parameter Theories where the deterministic measurement functions $A(\lambda, \mathbf{a})$ and $B(\lambda, \mathbf{b})$ are replaced by probabilistic functions. One then finds that the Bell's inequalities still hold, and that the conflict does not disappear. It is therefore generally accepted that the deterministic character of the formalism is not the reason for the conflict.

The most important ingredient, stressed by Bell in all his papers, is the local character of the formalism of subsection 4.I. We have indeed implicitly assumed that the result $A(\lambda, \mathbf{a})$ of the measurement at polarizer $\mathbf{l}$, does not depend on the orientation $\mathbf{b}$ of the remote polarizer II, and vice-versa. Similarly, it is assumed that the probability distribution $\rho(\lambda)$ (i.e. the way in which pairs are emitted) does not depend on the orientations $\mathbf{a}$ and $\mathbf{b}$. This locality assumption is crucial: Bell's Inequalities would no longer hold without it. It is indeed clear that the demonstration of 5.3 fails with quantities such as $A(\lambda, \mathbf{a}, \mathbf{b}) \operatorname{or} \rho(\lambda, \mathbf{a}, \mathbf{b})$.

To conclude, there are two ingredients that seem to be necessary to obtain Bell's inequalities, and thus to have a conflict with Quantum Mechanics :

- Distant correlations can be understood by introduction of supplementary parameters carried along by the separated particles, in the spirit of Einstein's ideas that separated objects have separated physical realities.

- The quantities $A(\lambda, \mathbf{a}), B(\lambda, \mathbf{b})$ and $\rho(\lambda)$ obey the Bell's locality condition, i.e. they do not depend on the orientations of the distant polarizers.

(3) For a simple demonstration, see for instance [18], available at http://tel.archives-ouvertes.fr/?langue=en 


\subsection{A Gedankenexperiment with variable analyzers: Bell's locality as a consequence of relativistic causality}

In static experiments, in which the polarizers are held fixed for the whole duration of a run, the Locality Condition must be stated as an assumption. Although highly reasonable, this condition is not prescribed by any fundamental physical law. To quote Bell [13]: "the settings of the instruments are made sufficiently in advance to allow them to reach some mutual rapport by exchange of signals with velocity less than or equal to that of light". In that case, the result of the measurement at polarizer I could depend on the orientation of the remote polarizer II, and vice-versa. The Locality Condition would no longer hold, nor would Bell's Inequalities. Bell thus insisted upon the importance of "experiments of the type proposed by Bohm and Aharonov [12], in which the settings are changed during the flight of the particles". In such a timing-experiment, the locality condition would become a consequence of Einstein's relativistic Causality that prevents any faster-than-light influence.

As shown in a 1975 proposal $[19,20]$, it is sufficient to switch each polarizer between two particular settings (a and a' for I, $\mathbf{b}$ and $\mathbf{b}$ ' for II), to test experimentally a very large class of Supplementary Parameters Theories: those obeying Einstein's relativistic Causality. In such theories, the response of polarizer I at time $t$, is allowed to depend on the orientation b (or b') of polarizer II at times anterior to $t-L / c$ ( $L$ is the distance between the polarizers and $c$ the velocity of light). A similar retarded dependence is considered for the probability distribution $\rho(\lambda)$, i.e. the way in which pairs are emitted at the source.

On the other hand, one can show [18] that the polarization correlations predicted by Quantum Mechanics depend only on the orientations $\mathbf{a}$ and $\mathbf{b}$ at the very time of the measurements, and do not involve any retardation terms. For a suitable choice of the set of orientations ( $\left.\mathbf{a}, \mathbf{a}^{\prime}, \mathbf{b}, \mathbf{b}^{\prime}\right)$ for instance the sets displayed on figure 4 the quantum mechanical predictions still conflict with generalized Bell's Inequalities.

\section{Experimental tests}

\section{I From the Gedankenexperiment to realistic experiments with entangled photons: a short history}

With Bell's inequalities, the debate on the possibility (or necessity) to complete Quantum Mechanics changed dramatically. It was no longer a matter of philosophical position (realism versus positivism), or of personal taste (Einstein vs. Bohr). It became possible to settle the question by an experiment. If one can produce pairs of photons in an EPR state, and measure the 4 coincidence rates $N \pm, \pm(\mathbf{a}, \mathbf{b})$ with detectors in the output channels of the polarizers (or Stern-Gerlach filters), one obtains directly, without any extra calibration the polarization correlation coefficient, for polarizers in orientations $\mathbf{a}$ and $\mathbf{b}$ : 


$$
E(\mathbf{a}, \mathbf{b})=\frac{N_{++}(\mathbf{a}, \mathbf{b})+N_{--}(\mathbf{a}, \mathbf{b})-N_{+-}(\mathbf{a}, \mathbf{b})-N_{-+}(\mathbf{a}, \mathbf{b})}{N_{++}(\mathbf{a}, \mathbf{b})+N_{--}(\mathbf{a}, \mathbf{b})+N_{+-}(\mathbf{a}, \mathbf{b})+N_{-+}(\mathbf{a}, \mathbf{b})}
$$

By performing four measurements of this type in orientations (a, b), (a, $\left.\mathbf{b}^{\prime}\right),\left(\mathbf{a}^{\prime}, \mathbf{b}\right)$ and $\left(\mathbf{a}^{\prime}, \mathbf{b}^{\prime}\right)$, one obtains a measured value $S_{\exp }\left(\mathbf{a}, \mathbf{a}^{\prime}, \mathbf{b}, \mathbf{b}^{\prime}\right)$ for the quantity defined in equation (19). Choosing a situation where Quantum Mechanics predicts that this quantity violates Bell's inequalities, we have a test allowing one to discriminate between Quantum Mechanics and any local supplementary parameter theory fulfilling the Bell's locality condition. If in addition we use a scheme with variable polarizers, we even test the more general class of Local Supplementary Parameters Theories where 'local' must be understood in the relativistic sense, i.e. as meaning 'space-like separated'.

Finding pairs of systems in an EPR-like entangled state is not easy. Pairs of spin 1/2 particles in a singlet state are an example, but none practical implementation has been done yet, although a somewhat related experiment has been done with protons [2I]. The disintegration of positronium in the singlet state leads to pairs of $\gamma$ photons in an EPR state, as emphasized by Bohm and Aharonov [12], but there is no polarizer for such $\mathrm{MeV}$ photons. Experiments must then rely on Compton scattering to calculate what would have been the result of a true polarization measurements if a polarizer would have existed [22]. After some conflicting results, experiments have been found in agreement with Quantum Mechanics [23, 24], but we are still far from a genuine test of Bell's inequalities.

A breakthrough was the proposal by Clauser et al. [14] to use pairs of optical photons, for which true polarizers do exist, and to show that pairs of photons emitted in well chosen atomic cascades would be in the EPR state of equation (2). This launched a first series of experiments, initially yielding conflicting results, but eventually giving clear results in favor of Quantum Mechanics [25, 26, 27]. These experiments were nevertheless still quite different from the ideal Gedankenexperiment scheme, and this is why I embarked into a new series of experiments, with the goal to achieve schemes closer and closer to the ideal scheme.

The development of a source of entangled photons of unprecedented efficiency, led to the publication of three tests of Bell's inequalities $[28,29,30]$, with the following new features:

- The polarizers were pulled at a distance of the source farther than the coherence length of the second photon, realizing a space-like separation between the measurement events.

- We implemented true polarization analyzers, with two output channels, in contrast to previous experiments realized with single channel polarizers where the second channel is lost. The scheme is then identical to the one of figure I, and one no longer depends on auxiliary calibrations and a supplementary hypothesis to test Bell's inequalities. The result was a spectacular violation of Bell's inequalities by more than 40 standard deviations, and a perfect agreement with the quantum mechanical predictions. 
- We implemented a scheme where the direction of analysis of polarization was changed while the photons were in flight, realizing a space-like separation between the choices of the measured observables, thus implementing a full separation in spacetime. Although not perfect since the choices of the directions of the polarizers were not fully random, this experiment was the first evidence of quantum non-locality in the relativistic sense.

All these three experiments gave a clear violation of Bell's inequalities, and a remarkable agreement with the prediction of Quantum Mechanics, in schemes closer and closer to the ideal Gedankenexperiment.

In the 1990's, progress in non-linear optical materials permitted to develop new types of sources of entangled photons, with the directions of emissions strictly correlated, in contrast to the previous sources based on atomic radiative cascades, where the momenta of the emitted photons are not correlated since the recoiling atom can accommodate momentum conservation for any emission directions. This permitted to inject the emitted photons in optical fibers, and to realize long distance tests of Bell's inequalities [3I, 32], leaving enough time to make a true random choice of the polarizers orientation [33]. Here again, the violation of Bell's inequalities was spectacular, and the agreement with Quantum Mechanics remarkable.

\subsection{Loophole-free tests}

A soon as it was realized that it was possible to test experimentally Bell's inequalities, in order to settle experimentally the question of the possibility to complete Quantum Mechanics, it was emphasized that real experiments could leave some loophole open for particular Supplementary Parameters Theories able to reproduce the experimental results. Three loopholes have been considered, and discussed in particular by Bell [34]:

I. The locality loophole.

2. The sensitivity loophole.

3. The free-will loophole.

The locality loophole refers to what was mentioned in subsections 6.2 and 6.3 , i.e., that in an experiment with static polarizers there might be an influence between the distant polarizers, and between the polarizers and the source. This loophole has been closed by the experiments of [29] and [33].

The sensitivity loophole refers to the fact that standard photon detectors have a limited quantum efficiency and miss a significant fraction of the photons. One can then develop supplementary parameters models in which the detectors select well chosen sub-ensembles of photons, to mimic Quantum Mechanics. In order to test Bell's inequalities with such experimental results, one must use a supplementary hypothesis, the 'fair sampling assumption', that states that the detected photons are a fair representative of the ensemble of photons, 
without any bias. Recent experiments using new types of high sensitivity detectors have closed that loophole [35, 36].

The free-will loophole is different in nature. It is based on the fact that it might happen that the apparently independent random choices of the settings of the distant polarizers are in fact determined by some cause in the backward light cone of the two choices. One could then have a local (in the relativistic sense) Supplementary Parameters Theory rendering an account of results violating Bell's inequalities. Following Bell, I doubt that there is any possibility to close that loophole, which could be used, as a matter of fact, to interpret anything happening in the world, since it amounts to accepting that things have been determined long ago (at the Big Bang?). A discussion exists, however, about the following question: is the randomness of a decision made by a human operator, supposed to have a full free will, similar in nature to the randomness of a 'Swiss lottery machine' (the favorite example of Bell) or any device we think really random?

\section{The emergence of quantum information}

After the experimental observation of the violation of Bell's inequalities, it could be thought that it was the end of the story. But in fact some physicists, in particular Feynman [37], realizing that entanglement is definitely different from wave particle duality, proposed to use it for new applications, and laid the ground for a new field of research, quantum information [38]. Quantum information involves totally new ways of transmitting and processing information, such as quantum cryptography, quantum teleportation, quantum computing, and quantum simulation. If these new methods become practical and available on a large scale, they may well change our society as deeply as the inventions of the transistor, the integrated circuits, and the laser, i.e. the fruits of the first quantum revolution, led our society into the Information and Communication society.

\section{I Quantum cryptography}

Cryptography is the science of encoding and/or transmitting a secret message without its being read/understood by a third party. Both encoding and code-breaking have progressed due to advances in mathematics and to the ever-increasing power of computers. When contemplating the continuing progress of encrypting and code breaking over ages, it seems clear that the security of an encrypted transmission can be assured only under the hypothesis that the adversary (who is trying to break the code) has neither more advanced mathematics nor more powerful computers than the sender and intended receiver.

By contrast, in quantum cryptography, the security of a transmission rests on the fundamental physical laws at work in Quantum Mechanics. There, it is possible to detect an eavesdropper by using the trace that is necessarily left by him/her $[39,40]$, since in quantum physics all measurements perturb the system in some way. In quantum cryptography one can check the absence of such a trace, and then be certain that the message (or more precisely an encoding key) has passed without having been read by a spy. 
Many demonstrations of quantum cryptography have been carried out, and commercial systems are already available and experimented in commercial (banking) or government (elections) activities.

\subsection{Quantum computing}

In the early 1980's, the fundamental assumption in information theory - that all computers are conceptually equivalent - started to be challenged. Several scientists, for instance R. Landauer or D. Deutsch, suggested that if one had a quantum computer, one could implement radically new algorithms to perform certain tasks. A breakthrough happened in 1994 when P. Shor showed that a quantum computer should allow one to factor large numbers in times much shorter than with conventional methods. Factorization belongs to a class of problems (complexity class) whose solution (with classical computers) requires a time super-polynomial in the size of the problem (that is, the time needed grows faster than any power of the number of digits in the number to be factored). With a quantum computer running the Shor's algorithm, on the other hand, the computation time would only grow as a power of the size of the number. This discovery had considerable conceptual implications, since it showed that contrary to what had been thought previously, the complexity class of a problem was not independent of the type of machine used. It also was the starting point of an immense experimental effort worldwide, aiming at realizing a quantum computer able to implement quantum algorithms such as the one of Shor.

Several groups have started to develop the basic elements of a quantum computer: quantum bits, and quantum gates. A quantum logic gate performs basic operations on quantum bits or "qubits", just as an electronic logic gate manipulates ordinary bits. However, in contrast to normal bits, which can take only one of the two values 0 and I, quantum bits can be put in a superposition of 0 and I. A quantum logic gate must thus be capable of combining two quantum bits to produce an entangled state which is the superposition of the four possible combinations $(0,0),(0, I),(I, 0),(I, I)$, of the two qubits basic states. It is the possibility to work with such entangled states that opens new and incommensurate possibilities as compared to the classical algorithms. To give a flavor of it, let us notice that if one entangles 10 qubits, the number of combinations of 0 and I states is now $2^{10}=1024$, while for 20 entangled qubits it is $2^{20}=$ about I million, etc. It means that with a limited number of qubits, constituting a quantum register, one can in principle store a huge amount of information, and that any operation acting on an entangled state will process a huge quantity of information, realizing a kind of massively parallel computing.

Experimental research on quantum gates is extremely active, and has already obtained important results. Many approaches are being explored, with a diversity of physical realizations of qubits, including atoms, ions, photons, nuclear spins, Josephson junctions, RF circuits... For all these systems there are large unknowns. A universal quantum computer would rely on the ability to entangle hundreds of thousands of quantum bits, and perform thousands of operations before decoherence disrupts the quantum register. Decoherence results from the interaction with the outside world, and its effect is to wash out entanglement, putting previously entangled objects into a state where they behave as separated objects. The scalability to a large number of entangled qubits may turn out to be overwhelmingly 
difficult since it is generally observed that decoherence dramatically increases when the number of entangled particles increases. An entire community of experimentalists and theorists is engaged in that quest. Understanding and reducing the effects of decoherence may well be the key question facing quantum computation, as a technological revolution. But even in the absence of an efficient quantum computer, the idea of quantum computation is certainly a milestone in computation science.

\subsection{Quantum simulation}

In contrast to quantum computing with quantum gates and qubits, another kind of quantum computing is already operational, that is quantum simulation. Quantum simulation is in fact what was primarily suggested in the 1982 Feynman's paper [37], often considered as the starting point of quantum information. In this paper, Feynman shows first that it is absolutely impossible but to store in a classical computer a quantum state of many entangled quantum systems, since this would demand a number of bits larger than the number of atoms in the universe. He then concludes that the only support for such huge quantity of information is a quantum system involving many entangled elementary quantum systems. A quantum computer made of many entangled quantum bits would be such a system. But there is another possibility, which has already led to several experimental implementations. It consists of considering a situation difficult to investigate directly, for instance many entangled electrons in a material, and simulating it with a system similar but offering more possibilities of study, such as many ultra-cold atoms in a potential synthesized with laser beams.

A first example is the case of electrons in a perfect crystal, i.e. in a perfect periodic potential. One can simulate such a situation by placing many ultra-cold atoms (atoms with a motion perfectly controlled at the quantum level) in a potential constituted of laser standing waves, whose intensity is modulated in an absolutely perfect periodic way along the three dimensions of space. This realizes a perfect lattice of potential wells where the atoms may be trapped. The cold atoms system has two main advantages. Firstly, one has many observation tools allowing experimentalists to directly observe the atoms and record their distribution in space, or the distribution of their velocities. Secondly, one can change parameters such as, for instance, the height of the barrier between neighbor trapping sites, in contrast to the case of electrons in a piece of material, where the parameters are given by the very nature of the material and can hardly be modified. By lowering the barriers between the sites where atoms were trapped, experimentalists could observe a transition from a situation where the atoms are fixed to a situation where they can propagate freely. This would correspond to a transition from an insulating to a supra-conducting state in the case of electrons, and such a quantum transition called 'a Mott transition' had been predicted decades ago, but never observed directly.

Another example is a situation where the atoms are plunged in a disordered potential realized with laser beams passed through a scattering plate. The intensity varies randomly in space, achieving a disordered potential that we can describe accurately with the tools of statistical optics. This has allowed us to observe another emblematic phenomenon of condensed matter physics, Anderson localization. This fully quantum phenomenon was 
predicted more than fifty years ago. The prediction was that when the randomness of the potential is large enough (or equivalently the density of impurities in a material is large enough), the motion of the particles (the electrons in a material) would not only be hindered but even totally stopped, due to a quantum interference between the many multiple-scattering paths. This is again a quantum phase transition, never observed directly with electrons in materials, which can be directly observed and studied with ultra-cold atoms.

To describe such condensed matter situations, only idealized theoretical models exist, and it often happens that we do not know exact solutions for these models. Quantum simulators allow one to implement these models, explore their solutions by changing the parameters, and check whether some of these solutions correspond to the observed phenomena.

\section{The Bohr-Einstein discussion: visionary}

For a long time, it was considered that the discussion between Einstein and Bohr on EPR was nothing else than a philosophical discussion, without any consequence on the practical way of doing physics. We know that the situation changed with the discovery by Bell that taking Einstein's point of view led to a conflict with Quantum Mechanics, but it is clear that it took a long time for this discovery to be recognized by a majority of physicists. Feynman gives us a remarkable example of this difficulty. In the early 1960's, in his famous 'Lectures on Physics' [4I], he writes that all the 'mystery' of Quantum Mechanics is in the wave-particle duality of a particle able to interfere with itself, and he considers that there is nothing special in the EPR situation: "This point was never accepted by Einstein... it became known as the 'Einstein-Podolsky-Rosen paradox'. But when the situation is described as we have done it here, there doesn't seem to be any paradox at all..." It took another 20 years for Feynman to realize that there is another quantum mystery, and to write [37]: "We always have had a great deal of difficulty in understanding the world view that Quantum Mechanics represents... It has not yet become obvious to me that there is no real problem... I've entertained myself always by squeezing the difficulty of Quantum Mechanics into a smaller and smaller place, so as to get more and more worried about this particular item. It seems to be almost ridiculous that you can squeeze it to a numerical question that one thing is bigger than another. But there you are - it is bigger..." What Feynman is alluding to is nothing else than the violation of Bell's inequalities, and the weirdness of entanglement.

It thus seems clear that the revolutionary nature of entanglement between several quantum objects took a very long time to be fully appreciated. But it seems just as clear that Bohr and Einstein had understood it in 1935, and it is a fact that for the rest of their lives their discussions about Quantum Mechanics centered on that point. Here again we can acknowledge that these two giants were far ahead of their contemporaries (with the exception of Schrödinger). Einstein because he discovered entanglement, and understood that it was different in nature from wave particle duality. Bohr because he had the correct intuition, thirty years before it was demonstrated by Bell, that adopting Einstein's position would lead to an inconsistency with Quantum Mechanics. At this time of celebration of Niels Bohr, it is good to notice that he was among the few physicists who had not underestimated the EPR discovery. 


\section{References}

[I] Planck, M.: Law of energy distribution in normal spectra. Annalen der Physik 4, pp. 553-563 $(|90|)$.

[2] Einstein, A.: Generation and conversion of light with regard to a heuristic point of view. Annalen Der Physik 17, n 6, pp. 132-148 (1905).

[3] Millikan, R.: Albert Einstein on his 70th Birthday. Reviews of Modern Physics 21, n 3, pp. 343-345 (1949).

[4] Bohr, N.: On the Constitution of Atoms and Molecules. Philosophical Magazine 26, pp. I-25 (19|3).

[5] Bohr, N.: On the Constitution of Atoms and Molecules. Philosophical Magazine 26, pp. 476-502 (19/3).

[6] Bohr, N.: On the Constitution of Atoms and Molecules. Philosophical Magazine 26, pp. 857-875 (1913).

[7] Jammer, M.: Philosophy of Quantum Mechanics. The interpretations of quantum mechanics in historical perspective. John Wiley \& Sons, 1974.

[8] Einstein, A., Podolsky, B., and Rosen, N.: Can quantum-mechanical description of physical reality be considered complete? Physical Review 47, n 10, pp. 777- 780 (1935).

[9] Schrödinger, E.: Discussion of probability relations between separated systems. Proceedings of the Cambridge Philosophical Society 31, pp. 555-563 (1935).

[I0] Bohr, N.: Can quantum-mechanical description of physical reality be considered complete? Physical Review 48, n 8, pp. 696-702 (1935).

[II] Bohm, D.: Quantum Theory. Dover Publications, 1951.

[12] Bohm, D., and Aharonov, Y.: Discussion of Experimental Proof for the Paradox of Einstein, Rosen, and Podolsky. Physical Review 108, n 4, pp. 1070-1076 (1957).

[13] Bell, J. S., et al.: On the Einstein-Podolsky-Rosen paradox. Physics I, n 3, pp. 195-200 (1964).

[14] Clauser, J. F., Horne, M. A., Shimony, A., and Holt, R. A.: Proposed experiment to test local hidden-variable theories. Physical Review Letters 23, n 15, pp. 880-884 (1969).

[15] Wenger, J., Hafezi, M., Grosshans, F., Tualle-Brouri, R., and Grangier, P.: Maximal violation of Bell inequalities using continuous-variable measurements. Physical Review A 67, n I, p. 012105 (2003).

[16] Schilpp, P. A.: Albert Einstein: philosopher-scientist. Vol. I. Open Court, 1970. 
[17] Born, M., Einstein, A., and Born, I.: The Born-Einstein Letters: correspondence between Albert Einstein and Max and Hedwig Born from 1916 to 1955 with commentaries by Max Born. Translated by Irene Born. Basingstoke, Macmillan Press, 1971.

[18] Aspect, A.: Three experimental tests of Bell inequalities by the measurement of polarization correlations between photons. PhD Thesis, vol. 2674, 1983.

[19] Aspect, A.: Proposed experiment to test separable hidden-variable theories. Physics Letters A 54, n², pp. 117-1।8 (1975).

[20] Aspect, A.: Proposed experiment to test nonseparability of quantum-mechanics. Physical Review D 14, n 8, pp. 1944-1951 (1976).

[2I] Lamehi-Rachti, M., and Mittig, W.: Quantum mechanics and hidden variables: A test of Bell's inequality by the measurement of the spin correlation in low- energy proton-proton scattering. Physical Review D 14, no 10, p. 2543-2555 (1976).

[22] Kasday, L., Ullman, J., and Wu, C.: Angular correlation of compton-scattered annihilation photons and hidden variables. II Nuovo Cimento B Series II 25, n², pp. 633-66I (1975).

[23] Bruno, M., Dagostino, M., and Maroni, C.: Measurement of linear polarization of positronannihilation photons. Nuovo Cimento della Societa Italiana di Fisica B-General Physics Relativity Astronomy and Mathematical Physics and Methods 40, n I, pp. I43-I52 (1977).

[24] Wilson, A., Lowe, J., and Butt, D.: Measurement of the relative planes of polarization of annihilation quanta as a function of separation distance. Journal of Physics G: Nuclear Physics 2, n०9, p. 613 (1976).

[25] Clauser, J. F.: Experimental investigation of a polarization correlation anomaly. Physical Review Letters 36, n²1, pp. 1223-1226 (1976).

[26] Freedman, S. J., and Clauser, J. F.: Experimental test of local hidden-variable theories. Physical Review Letters 28, n 14, pp. 938-941 (1972).

[27] Fry, E. S., and Thompson, R. C.: Experimental test of local hidden-variable theories. Physical Review Letters 37, n 8, pp. 465-468 (1976).

[28] Aspect, A., Grangier, P., and Roger, G.: Experimental tests of realistic local theories via Bell's theorem. Physical Review Letters 47, n 7, pp. 460-463 (1981).

[29] Aspect, A., Dalibard, J., and Roger, G.: Experimental test of Bell inequalities using time-varying analyzers. Physical Review Letters 49, n 25, pp. 1804-1807 (1982).

[30] Aspect, A., Grangier, P., and Roger, G.: Experimental realization of Einstein- PodolskyRosen-Bohm Gedankenexperiment- a new violation of Bell inequalities. Physical Review Letters 49, n², pp. 91-94 (1982). 
[3I] Tapster, P., Rarity, J., and Owens, P.: Violation of Bell inequality over $4 \mathrm{~km}$ of optical-fiber. Physical Review Letters 73, no 14, pp. 1923-1926 (1994).

[32] Tittel, W., Brendel, J., Zbinden, H., and Gisin, N.: Violation of Bell inequalities by photons more than 10 km apart. Physical Review Letters 8I, n 17, pp. 3563-3566 (1998).

[33] Weihs, G., Jennewein, T., Simon, C., Weinfurter, H., and Zeilinger, A.: Violation of Bell's inequality under strict Einstein locality conditions. Physical Review Letters 8I, n²3, pp. 5039-5043 (1998).

[34] Bell, J. S.: Speakable and Unspeakable in Quantum Mechanics: Collected Papers on Quantum Philosophy. Cambridge University Press, 2004.

[35] Giustina, M., Mech, A., Ramelow, S., Wittmann, B., Kofler, J., Beyer, J., Lita, A., Calkins, B., Gerrits, T., Nam, S., Ursin, R., and Zeilinger, A.: Bell violation using entangled photons without the fair-sampling assumption. Nature 497, pp. 227-230 (2013).

[36] Christensen, B. G., McCusker, K. T., Altepeter, J. B., Calkins, B., Gerrits, T., Lita, A. E., Miller, A., Shalm, L. K., Zhang, Y., Nam, S. W., Brunner, N., Lim, C. C. W., Gisin, N., and Kwiat, P. G.: Detection-Loophole-Free Test of Quantum Nonlocality, and Applications. Physical Review Letters III, 130406 (20I3).

[37] Feynman, R. P.: Simulating physics with computers. International Journal of Theoretical Physics 21, n 6-7, pp. 467-488 (1982).

[38] Nielsen, M. A., and Chuang, I. L.: Quantum Computation and Quantum Information. Cambridge University Press, 2010.

[39] Bennett, C. H., Brassard, G., and Ekert, A. K.: Quantum cryptography. Scientific American 267, n4, pp. 50-57 (1992).

[40] Ekert, A. K.: Quantum cryptography based on Bell theorem. Physical Review Letters 67, n॰ 6, pp. 661-663 (1991).

[4I] Feynman, R. P.: Lectures on Physics. Addison-Wesley, 1963. 